\title{
ROCK inhibitor, Y-27632, reduces FBS-induced structural alteration in organ-cultured mesenteric artery
}

Yang Hoon Huh ${ }^{1,2}$, Hee-Seok Kweon ${ }^{1 *}$ and Toshio Kitazawa ${ }^{2,3^{*}}$

\begin{abstract}
Background: Chronic treatment with fetal bovine serum (FBS) causes gradual vasoconstriction, vascular wall thickening, and contractility reduction in organ-cultured vascular tissues. We have previously demonstrated that Rho-associated kinase (ROCK) inhibitors prevent the functional alterations of small arteries in response to the FBS treatment. Here, we tested a further hypothesis that the chronic inhibition of ROCK has a protective effect on FBS-induced structural alterations.

Methods: To verify the new hypothesis, the rabbit mesenteric arterial rings were cultured in FBS-supplemented culture medium with or without Y-27632, a reversible ROCK inhibitor and then western blot,

immunohistochemistry, apoptosis assay, and electron microscopy were performed using organ-cultured arterial rings.

Results: Chronic treatment with Y-27632 maintained the arterial diameter by preventing FBS-induced gradual arterial constriction during organ culture. Y-27632 also reduced the apoptosis and the loss of contractile myosin and actin filaments of smooth muscle cells. In addition, Y-27632 protected the morphological integrity between the endothelial cell layer and smooth muscle cell layer by preventing endothelial cell detachment and platelet endothelial cell adhesion molecule (PECAM) expression decrement.

Conclusions: Chronic ROCK inhibition provides protective effects against FBS-stimulated structural in addition to functional alterations of vascular smooth muscle cells and endothelial cells. These results strongly suggest that the RhoA/ROCK signaling is crucial for maintaining the structural and functional phenotypes of vasculature, and hence, chronic ROCK inhibition may provide protective effects on excessive growth factor-related vascular diseases including hypertension and atherosclerosis.
\end{abstract}

Keywords: Cardiovascular disease; Artery; Fetal bovine serum; Organ culture; RhoA/ROCK signaling

\section{Background}

Sustained vasoconstriction and vascular remodeling play important roles in the pathogenesis of vascular diseases such as hypertension, vasospasm, and atherosclerosis (Schwartz 1997; Noma et al. 2006). Recent studies provided better understanding of the pathophysiological mechanisms involved in vascular dysfunction. However, cardiovascular disease is the number one cause of death throughout

\footnotetext{
* Correspondence: hskweon@kbsi.re.kr; kitazawa.toshio@gmail.com 'Division of Electron Microscopic Research, Korea Basic Science Institute, 169148 Gwahangno, Yuseong-gu, 305-806, Daejeon, Republic of Korea 2Boston Biomedical Research Institute, 64 Grove St, 02472, Watertown, MA, USA

Full list of author information is available at the end of the article
}

the world (World Health Organization 2011). Further studies are needed in order to clarify the mechanism that causes vasoconstriction and vascular remodelinginduced cardiovascular disease.

Ras homolog gene family member A (RhoA) acts as a molecular switch that regulates smooth muscle contraction (Brown et al. 2006), and Rho-associated kinase (ROCK), a RhoA effector, contributes to various physiological functions in blood vessels including endothelial and smooth muscle cells (Noma et al. 2006). Therefore, the RhoA/ROCK signaling pathway is proposed to play a pivotal role in diverse cellular functions, including expression and activity of endothelial nitric oxide synthase, 
vascular smooth muscle contraction, actin cytoskeleton organization, cell adhesion and motility, proliferation, and hypertrophy in smooth muscle cells (Noma et al. 2006; Somlyo and Somlyo 2003; Gerthoffer 2007). Although deletion of either ROCK1 or 2 causes, respectively, embryonic and postnatal death in mouse models, abnormal upregulation of the RhoA/ROCK signaling pathway induces various vascular dysfunctions (Schwartz 1997; Félétou and Vanhoutte 2006; Cicek et al. 2013), which suggests that control of RhoA/ROCK signaling pathway or RhoA and/or ROCK expression itself is a useful therapeutic target in cardiovascular diseases (Noma et al. 2006; Cicek et al. 2013; Shimokawa and Takeshita 2005; Loirand et al. 2006).

To address the underlying mechanisms by which the upregulation of RhoA/ROCK signaling causes abnormal contractility of the vascular smooth muscle and following vascular disease, various studies were carried out by the use of an animal model and isolated or cultured cell model. In an animal model, the beneficial effect of ROCK-inhibiting compounds including Y-27632 has been reported on various cardiovascular diseases such as hypertension, coronary and cerebral vasospasm, and pulmonary arterial hypertension (Uehata et al. 1997; Shimokawa and Rashid 2007). In vitro cell culture models also have been used extensively to elucidate the molecular mechanism governed by RhoA/ROCK signaling with finely tuned culture conditions (Chen et al. 2010; Singh et al. 2011; Pagiatakis et al. 2012). However, both approaches have particular limitations. For in vivo animal models, the underlying mechanisms on the effects of ROCK inhibitors are difficult to identify because disease conditions are difficult to control and many factors can be involved. In the case of in vitro cell culture models, it is difficult to regenerate the contractile machinery in smooth muscle cells and the smooth muscle cell-endothelial cell interactions. In addition, smooth muscle cells in culture rapidly transform their phenotypes from contractile to synthetic (Somlyo and Somlyo 2003; Owens et al. 2004; Woodsome et al. 2006). Thus, organ culture of the vasculature has been used as an alternative method that can complement the limitations of animal- or cultured cell-based studies for investigating the chronic treatment effects of various inhibitors in arterial tissues (Ozaki and Karaki 2002; Thorne and Paul 2003; Huh et al. 2011).

Recently, using an organ culture model, we have reported that the ROCK inhibitor Y-27632 preserved the small mesenteric arterial rings from 1-week fetal bovine serum (FBS)-supplemented culture-induced functional abnormalities, such as gradual arterial constriction, wall thickening, reduced contractile activity, downregulation of endothelial nitric oxide synthase (eNOS), reduction of endothelium-induced relaxation, and increased ROCKspecific myosin phosphatase targeting subunit 1 (MYPT1)
Thr 853 phosphorylation (Huh et al. 2011). In this report, we further expanded the previous functional study to a structural study. We tested whether inhibition of ROCK mitigates the effect of the growth stimulant FBS on the structure of differentiated smooth muscle and endothelial cells in peripheral arteries, which primarily regulate blood pressure. Here, we report that the ROCK inhibitor Y27632 prevents the detachment and loss of the endothelial cell layer, the disappearance of contractile thick and thin filaments, and apoptosis and necrosis. These results strongly suggest that the RhoA/ROCK signaling is crucial for maintaining the structural and functional phenotypes of the vasculature and hence has the potential to be a therapeutic target in cardiovascular diseases.

\section{Methods}

\section{Animals and mesenteric artery preparation}

All animal procedures for the isolation of arteries from rabbits were approved by the Animal Care and Use Committees of the Boston Biomedical Research Institute. The detailed procedures for isolation of arterial rings and organ culture have been described previously (Huh et al. 2011). Briefly, the rabbits were euthanized with an overdose of halothane. After thoracotomy, the mesenteric arteries were isolated and surrounding fat and fluffy connective tissues were discarded. To choose which segment in a mesenteric arterial tree is apt to conduct the morphofunctional studies using organ culture system, we first analyzed the gross morphology and protein expression of the main artery and first and second branches of the rabbit mesenteric artery. In the hematoxylin and eosin-stained low- and high-magnification images, no morphological difference between the main and branched mesenteric artery was noted except for the number of smooth muscle cell layers in the section (Figure 1A). Moreover, the contractile protein expressions related to the RhoA/ROCK signaling pathway were quite similar (Figure 1B,C). Based on these data, we chose the first branches of the mesenteric artery for organ culture because the inner and outer diameters were more uniform than the main artery and second branches of the mesenteric artery regardless of the region.

\section{Organ culture procedure}

To set up the organ culture condition, the first branches of the mesenteric artery were cut into rings $(0.5 \sim 0.6 \mathrm{~mm}$ in outer diameter and $0.75 \mathrm{~mm}$ in length). The arterial rings were then placed in a silicone elastomer dish containing $4 \mathrm{ml}$ of Dulbecco's modified Eagle's medium (DMEM) supplemented with $50 \mathrm{U}$ penicillin, $50 \mu \mathrm{g} / \mathrm{ml}$ streptomycin, and 10\% FBS (Sigma-Aldrich Co., St. Louis, MO, USA; cat\# F4135) with or without $10 \mu \mathrm{M}$ Y-27632. The arterial rings were maintained at $37^{\circ} \mathrm{C}$ with $5 \% \mathrm{CO}_{2}$ for 7 days. The culture medium was changed every 2 days. 
A

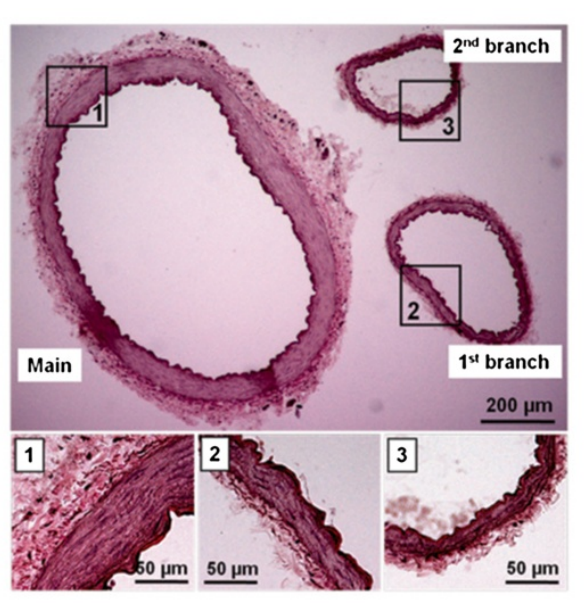

B

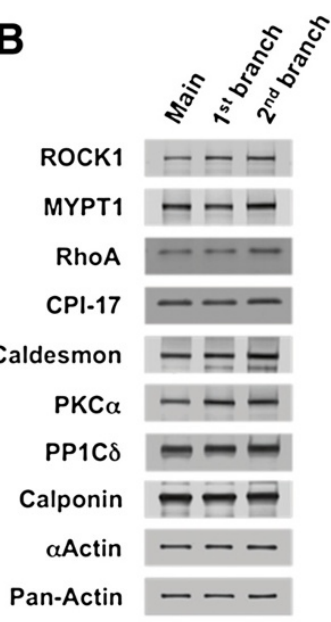

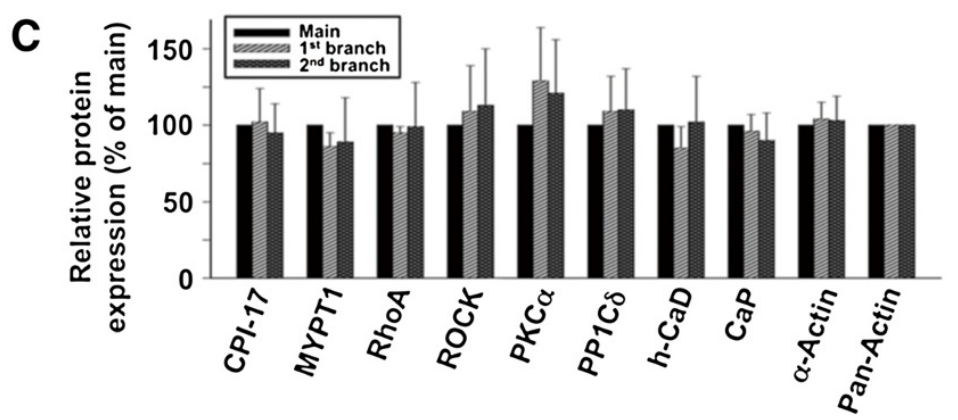

Figure 1 Gross morphology and contractile protein expression in the rabbit mesenteric artery. (A) To compare the gross morphology, the isolated main artery and first and second branches of the rabbit mesenteric artery were embedded in the same OCT block, and the collected cryosections were stained with hematoxylin and eosin. The high-magnification images of the boxed regions $(1,2,3)$ are displayed below. (B, C) Representative immunoblot images and a quantitative summary for relative contractile protein expression $(n=4)$. The protein expressions of the main artery and first and second branches of the mesenteric artery were compared after matching the pan-actin expression. For actins, protein extracts that were diluted tenfold were loaded.

During the culture, each ring was loosely laid and placed on the silicone elastomer and secured by a pin at the center of the lumen without stretching (see Figure 2A). Once or twice a day during culture, the medium was gently shaken and the rings were moved around the supporting pin to prevent it from attachment to the silicone dish and subsequent cell migration.

\section{Artery immunohistochemistry}

For immunohistochemistry analysis, the stretched arterial rings on the force transducer were fixed with $4 \%$ paraformaldehyde in phosphate-buffered saline (PBS) solution for $30 \mathrm{~min}$ at $30^{\circ} \mathrm{C}$. Then, the pre-fixed rings were removed from the apparatus and further fixed with $4 \%$ paraformaldehyde for $2 \mathrm{~h}$ at $4^{\circ} \mathrm{C}$. After three washes in PBS, the rings were embedded in the Tissue-Tek optimal cutting temperature (OCT) compound (Fischer Scientific, Waltham, MA, USA), frozen in liquid nitrogen-cooled isopentane, and kept at $-80^{\circ} \mathrm{C}$ for future use. The cryostat sections $(10 \mu \mathrm{m})$ were collected on glass slides, washed with PBS to remove OCT compound, treated with $0.2 \%$
Triton X-100, and washed with PBS. The sections were then incubated in the blocking solution (PBS containing $10 \%$ goat serum, $5 \%$ milk, and $4 \%$ bovine serum albumin (BSA)) for $1 \mathrm{~h}$ at room temperature and treated with the primary antibody solution (PBS containing 0.1\% Tween-20 and $2 \% \mathrm{BSA}$ ) for overnight at $4{ }^{\circ} \mathrm{C}$ in humidified chambers. After rinsing with PBS containing 0.5\% BSA, the sections were treated with fluorescence-conjugated secondary antibody (Invitrogen, Inc., Carlsbad, CA, USA). After washing, the sections were counterstained with 4',6-diamidino-2-phenylindole (DAPI) and mounted with FluorSave mounting medium (Millipore Corp., Billerica, MA, USA). Epifluorescence images were captured with a Leica DMR fluorescence microscope and a Leica DC300F digital camera system (Leica Microsystems Ltd., Milton Keynes, UK).

\section{Immunoblotting}

Immunoblotting experiments were performed as previously described (Kitazawa et al. 2003). Briefly, the arterial rings were isolated from the main artery and first and second 


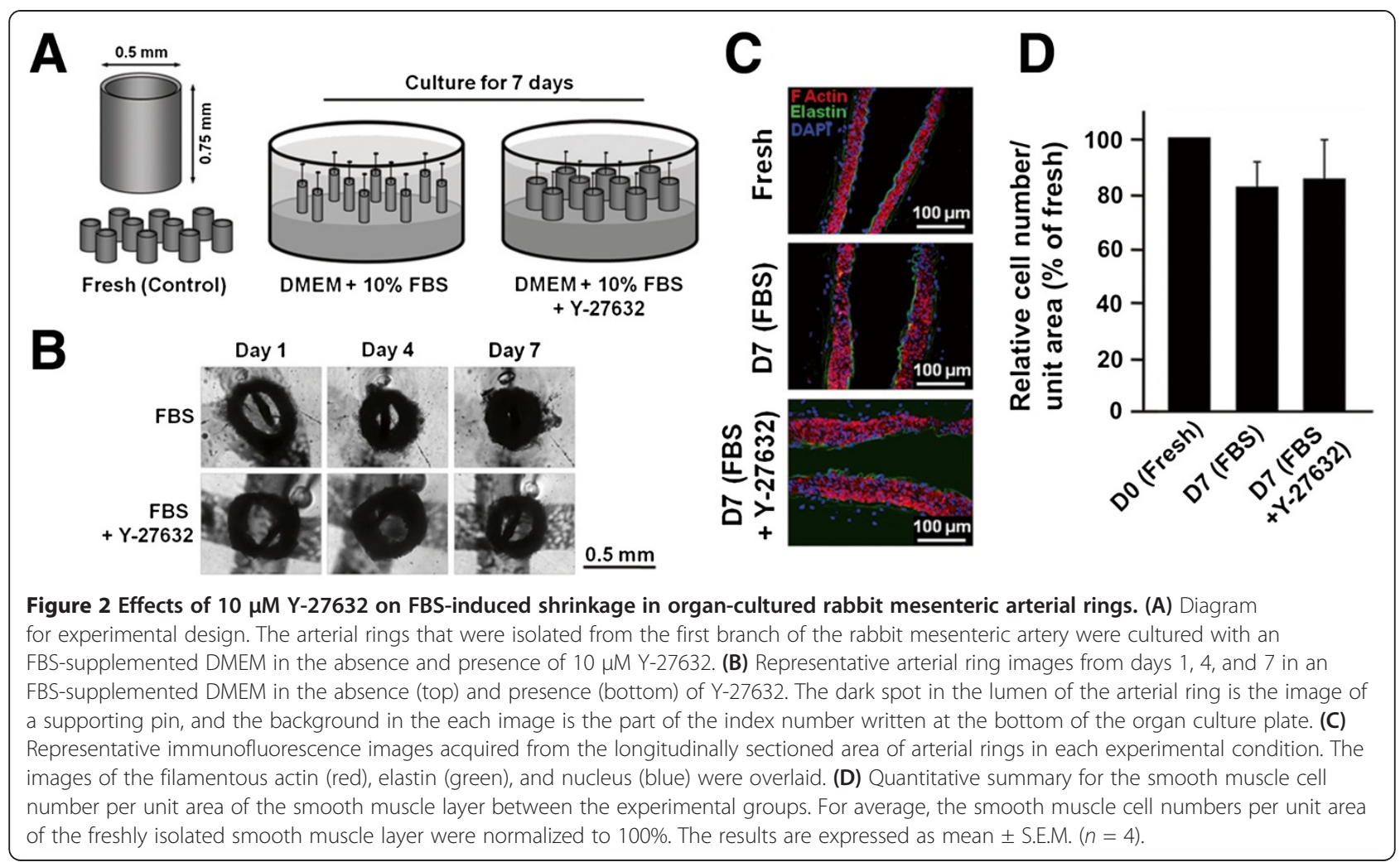

branches of the mesenteric artery, and they were quickly frozen in liquid nitrogen and kept in 10\% trichloroacetic acid (TCA)-acetone at $-80^{\circ} \mathrm{C}$ overnight. After the TCA treatment, the tissues were gradually warmed and washed in acetone at room temperature, and allowed to dry. The small dried rings were homogenized in a Laemmli sample buffer (LSB; with final concentrations of $62.5 \mathrm{mM}$ Tris, $1 \%$ SDS, $15 \%$ glycerol, $30 \mathrm{mM}$ dithiothreitol, and $0.005 \%$ bromophenol blue) using a glass-glass mini homogenizer. The homogenates were then centrifuged and the supernatants were collected. The total protein concentration was measured using a Coomassie Plus Protein Assay Reagent Kit (Pierce, Rockford, IL, USA) and adjusted to $2 \mathrm{mg} / \mathrm{ml}$ with LSB. For the actin, the samples were diluted tenfold with LSB. The proteins were separated on a $4 \%$ to $20 \%$ polyacrylamide gradient gel and then transferred to nitrocellulose membranes using a wet transfer method. The membranes were blocked in Tris-buffered saline (TBS) solution containing $0.05 \%$ Tween-20 and 5\% non-fat milk for $1 \mathrm{~h}$ at room temperature. After the treatment with the primary antibody solution, the membranes were incubated with alkaline phosphatase-conjugated secondary antibodies, and the bands were developed with alkaline phosphatase substrate (Sigma-Aldrich Co., St. Louis, MO, USA). The bands were scanned and analyzed using the IPLabGel image analyzing system (Signal Analytics, Vienna, VA, USA).

\section{Apoptosis detection by the TUNEL assay}

For the in situ apoptosis detection at the cellular level, we used the ApopTag red in situ apoptosis detection kit (Chemicon International, Temecula, CA, USA) for the terminal deoxynucleotide transferase dUTP nick end labeling (TUNEL) assay. Briefly, the cryostat sections $(10 \mu \mathrm{m})$ from the 7 days organ-cultured arterial rings with or without $10 \mu \mathrm{M}$ Y-27632 were obtained. The sections from the fresh mesenteric artery and the artery cultured in $1 \mu \mathrm{M}$ angiotensin II-containing medium for 7 days were used as the negative and positive control, respectively. The tissue sections were fixed in $1 \%$ paraformaldehyde for $2 \mathrm{~h}$ at $4^{\circ} \mathrm{C}$. After three washes in PBS (5 min each), the tissues were post-fixed with a mixture of ethanol and acetic acid (at a ratio of $2: 1$ ) at $-20^{\circ} \mathrm{C}$ for $5 \mathrm{~min}$ and washed three times ( $5 \mathrm{~min}$ each). The tissues were then incubated with the terminal deoxynucleotidyl transferase (TdT) enzyme in a humid atmosphere for $60 \mathrm{~min}$ at $37^{\circ} \mathrm{C}$. After incubation for $30 \mathrm{~min}$ with a rhodamine-labeled anti-digoxigenin conjugate and following counterstaining with DAPI, the sections were mounted with a FluorSave mounting medium (Millipore Corp., Billerica, MA, USA). Slides were viewed and imaged with a fluorescence microscope (Leica DMR) and a digital camera (Leica DC300F) system. 


\section{Transmission electron microscopy}

For transmission electron microscope (TEM) analysis, mesenteric arterial rings in different organ culture conditions were mounted on the force transducer setup and stretched to adjust the muscle length as described previously (Kitazawa et al. 2009). The fresh arterial rings were stretched to 1.2 times their original slack length and the organ-cultured rings were stretched to the same tension level as that of the fresh one. The stretched arterial rings were fixed for $30 \mathrm{~min}$ at $30^{\circ} \mathrm{C}$ in pre-warmed $0.1 \mathrm{M}$ sodium cacodylate buffer containing $2 \%$ glutaraldehyde, $2 \%$ paraformaldehyde, and $3.5 \%$ sucrose while they were stretched on the force transducer. Then, the pre-fixed rings were removed from the apparatus and further fixed in the same fixing solution for $2 \mathrm{~h}$ at $4^{\circ} \mathrm{C}$ with agitation. After three washes in $0.1 \mathrm{M}$ sodium cacodylate buffer (10 $\mathrm{min}$ each), the rings were post-fixed with $1 \%$ osmium tetroxide on ice for $2 \mathrm{~h}$ and washed three times (10 min each) with a $0.1 \mathrm{M}$ sodium cacodylate buffer. After gradual dehydration in an ethanol and propylene oxide series, the rings were then embedded in an Epon 812 mixture and polymerized in an oven at $60^{\circ} \mathrm{C}$ for $24 \mathrm{~h}$. The embedded blocks were then sectioned on an ultramicrotome with a diamond knife, and ultrathin sections were collected on collodion-coated copper grids. The sections were stained with $2.5 \%$ uranyl acetate $(7 \mathrm{~min})$ and Reynolds lead citrate (2 min) and were examined using a TEM (Technai $\mathrm{G}^{2}$ Spirit Twin, FEI, Hillsboro, OR, USA) at $120 \mathrm{kV}$.

\section{Antibodies}

The following primary antibodies were used in this study: polyclonal anti-MYPT1 (BabCO, Richmond, CA, USA, 1:5,000), anti-CPI-17 IgY (1:5,000), polyclonal anticaldesmon (from Dr. A. Wang of BBRI, 1:10,000), polyclonal anti-h-calponin (from Dr. E. Mabuchi of BBRI, 1:10,000), polyclonal anti-PKC $\alpha$ (Sigma, St. Louis, MO, USA, 1:1000), polyclonal anti-PP1C $\delta$ (from Dr. Eto of Thomas Jefferson University, 1:5,000), polyclonal antiROCK1 (Sigma, St. Louis, MO, USA, 1:2,000), monoclonal anti-RhoA (Santa Cruz Biotechnology, Dallas, Texas, USA, 1:1,000), polyclonal anti pan-actin (Sigma, St. Louis, MO, USA, 1:1,000), monoclonal anti $\alpha$-actin (Sigma, St. Louis, MO, USA, 1:5,000), and polyclonal anti-platelet endothelial cell adhesion molecule (PECAM)-1 (Santa Cruz Biotechnology, Dallas, Texas, USA, 1:1,000). The secondary antibody against chicken IgY was from Promega (Madison, WI, USA, 1:5,000). The anti-mouse and anti-rabbit IgG secondary antibodies $(1: 5,000)$ were from Chemicon (Billerica, MA, USA).

\section{Statistics}

The results are expressed as mean \pm S.E.M. of $n$ experiments. Statistical significance was evaluated with one-way ANOVA; $P<0.05$ was considered statistically significant.

\section{Results}

Effect of Y-27632 on the FBS-induced contraction of the organ-cultured artery

To examine the morphological changes in the arterial rings during the FBS-supplemented organ culture, the isolated arterial rings were cultured for 7 days with $10 \%$ FBS-supplemented DMEM with or without $10 \mu \mathrm{M}$ Y27632 (Figure 2A). Then, we obtained an image of each arterial ring every $24 \mathrm{~h}$ to analyze the changes in outer and inner diameters (i.e., thickness) of the arterial wall (Figure 2B). We first confirmed the previous findings (Huh et al. 2011) that the arterial rings organ-cultured in the $10 \%$ FBS medium in the absence of Y-27632, the inner diameter shrank to about half at day 4 , and the lumen was completely obstructed on day 7 (Figure 2B, top panel). On the other hand, $10 \mu \mathrm{M}$ Y-27632 markedly reduced the gradual shrinkage of the organ-cultured arterial rings and the changes in the arterial wall thickness (Figure 2B, bottom panel).

Then, we check precisely whether the shrinkage of arterial rings cultured with FBS in the absence of Y-27632 was mediated by smooth muscle cell migration to the culture plate from the tunica media possibly by the FBSmediated phenotypic change. All arterial rings used for the organ culture were fixed and double-stained with anti $\alpha$-actin antibody and DAPI, and the total cell number in the whole cross-sectional area of the tunica media was counted (Figure 2C). The total number of DAPI-stained nuclei in the tunica media of the arterial rings cultured for 7 days with FBS in the absence of Y-27632 (81 \pm $9 \%$ of fresh, $n=4)$ and in the presence of Y-27632 (85 \pm $14 \%$ of fresh, $n=4$ ) was not significantly different from that of the freshly isolated mesenteric artery (Figure 2D).

\section{Y-27632 effect on the maintenance of the endothelial cell and smooth muscle cell integrity in the organ-cultured artery}

We showed previously that Y-27632 prevented FBSinduced decrease in the eNOS mRNA expression and the acetylcholine (Ach)-induced relaxation (Huh et al. 2011). To examine whether Y-27632 also prevented FBS-induced structural alteration of the endothelial cell layer, the freshly isolated and organ-cultured mesenteric arteries that had been tested for Ach-induced relaxation were fixed and longitudinally (parallel to the longitudinal axis of the smooth muscle) sectioned for immunohistochemical analysis. Figure 3A shows the fluorescence images of the arterial section representing the anti-PECAM-stained endothelial cells, auto-fluorescencing elastin layer, DAPIstained nucleus, and merged images, respectively. In the freshly isolated mesenteric artery, one continuous PECAM layer (red, left image of top panel) was coincident with one side of the auto-fluorescencing elastin lines (green, left image of second panel), which suggests that the 

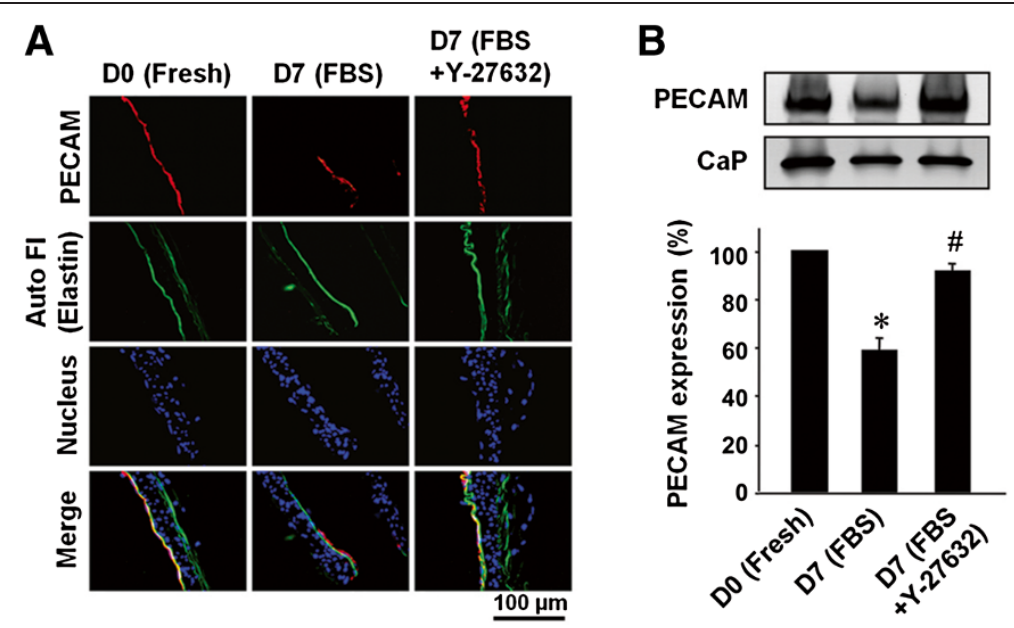

Figure 3 Effect of $10 \mu \mathrm{M}$ Y-27632 on the maintenance of endothelium integrity in organ-cultured mesenteric arteries. (A) Representative images of the endothelial layer that were immunostained with anti-PECAM (CD31) antibody in the longitudinal sections of freshly isolated and organ-cultured mesenteric arteries. To verify the morphological integrity of the endothelial cell layer, elastin autofluorescence (Auto Fl; green) and cell nucleus (DAPl; blue) were also captured with PECAM (red). (B) Representative immunoblot images of PECAM and calponin (as a loading marker) and a quantitative summary (lower) for the relative PECAM expression $(n=3)$. The asterisk represents a significant $(P<0.05)$ difference from that of the fresh mesenteric artery. The number sign represents a significant $(P<0.05)$ difference from that of the FBS-supplemented organcultured mesenteric artery in the absence of Y-27632.

endothelial cell layer fully covers the tunica intima and is tightly associated with the elastin layer (left image of bottom panel). By contrast, in the organ-cultured artery with FBS in the absence of Y-27632, approximately half of the endothelial cell layers were missing (middle image of top panel) and the remaining PECAM-positive layers were only loosely associated with the auto-fluorescencing elastin layer (middle image of bottom panel). This result suggests that considerable numbers of endothelial cells were already absent and the remaining layers were not tightly associated with the internal elastic layer. In the presence of Y-27632, however, majority of the endothelial cell layers except the small areas unstained to the PECAM (right image of top panel) were tightly adhered to the elastin layer along the luminal side of the vascular wall (right image of bottom panel).

To confirm the immunohistochemical results, we performed immunoblotting and determined the changes in the PECAM expression during organ culture. The PECAM expression in the organ-cultured artery without Y-27632 was significantly decreased to about half, whereas the PECAM expression was maintained at a level similar to that of the fresh mesenteric artery in the presence of $10 \mu \mathrm{M} \mathrm{Y-27632} \mathrm{(Figure} \mathrm{3B).} \mathrm{These} \mathrm{results} \mathrm{are} \mathrm{consistent}$ with the previous functional finding on ACh-induced relaxation (Huh et al. 2011).

\section{Y-27632 effect on the apoptotic and ultrastructural} degeneration in the organ-cultured mesenteric artery We showed previously that the artery organ-cultured with FBS for 7 days reduced both depolarization- and agonist-induced contractions and that Y-27632 significantly prevented the FBS-induced reduction of contraction (Huh et al. 2011). To test the hypothesis whether FBS treatment prompted and Y-27632 prevented cell death and/or phenotypic change during organ culture, we performed the apoptosis assay using the TUNEL method (Figure 4). To delineate the smooth muscle-containing tunica media, nuclei were counterstained with DAPI and the elastin autofluorescence was captured. Then, the apoptotic cells in the tunica media of each experimental condition were counted, respectively. The FBS-containing organ culture increased the number of TUNEL-positive apoptotic cells to $17 \pm 2 \%(n=7)$ of the total cells counted in the tunica media (second panel of Figure 4A), whereas Y-27632 limited apoptotic cells in the tunica media to $5 \pm 2 \%$ (third panel of Figure 4A) as compared to a negligible background level $(1 \pm 0 \%)$ in the freshly isolated mesenteric artery. We used the arterial rings that were cultured with $1 \mu \mathrm{M}$ angiotensin II (AT II) as a positive control sample (Best et al. 1999) to confirm the assay efficiency ( $n=7$; bottom panel of Figure 4A). Angiotensin II drastically increased the apoptotic cell fraction to $71 \pm 3 \%$ in the tunica media (Figure 4B).

Although RhoA and ROCK are upregulated and MYPT1 is phosphorylated in the chronic FBS organ culture conditions, the extent of contraction induced by agonists such as the $\alpha_{1}$-agonist is significantly lower after FBS treatment. This may be partly due to about $50 \%$ reduction in expression of the myosin light chain (MLC) and CPI-17 (Huh et al. 2011). We further tested whether the ultrastructural integrity of smooth muscle cells is 

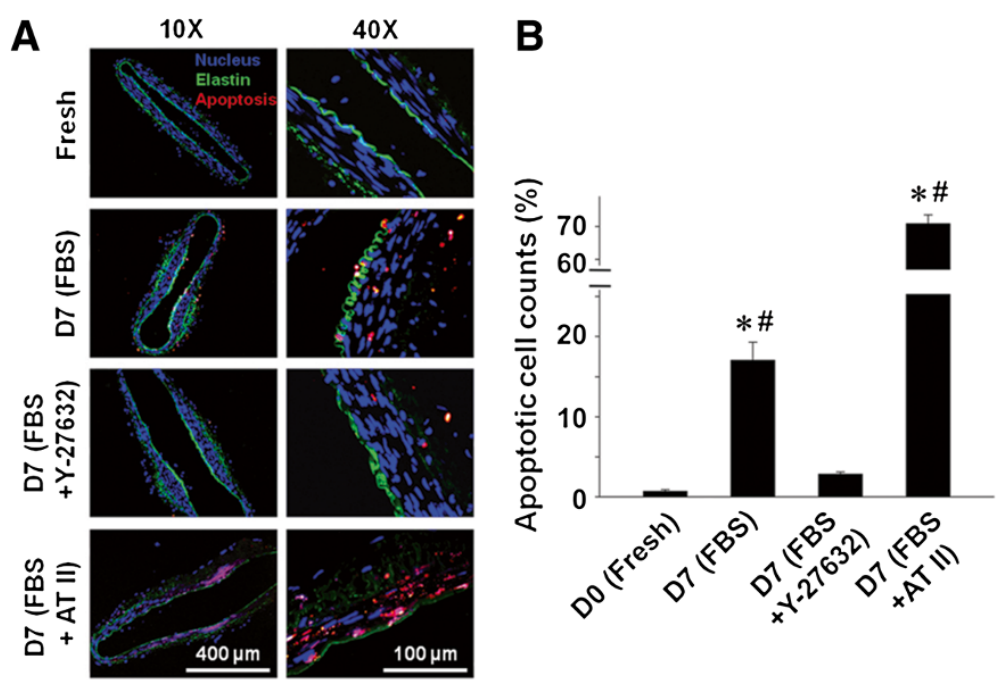

Figure 4 Effect of $10 \mu \mathrm{M} \mathrm{Y-27632}$ on the apoptotic degeneration of organ-cultured rabbit mesenteric arteries. (A) Cross-sections of the freshly isolated and organ-cultured mesenteric arteries were stained using the TUNEL method. The cell nuclei were counterstained with DAPI, and the elastin autofluorescence was captured to define the tunica media. To validate the TUNEL assay results, we used the arterial rings cultured with $1 \mu \mathrm{M}$ angiotensin II (AT II) as the positive control sample. The representative TUNEL (red) images merged with the DAPI (blue) and autofluorescence (green) images. (B) Statistical analysis of TUNEL data in the tunica media under each condition $(n=7)$. The asterisk represents a significant $(P<0.05)$ difference from that of the fresh mesenteric artery on D0. The number sign represents a significant $(P<0.05)$ difference from that of the FBS-supplemented organ-cultured mesenteric artery in the presence of Y-27632.

impaired with FBS treatment. The mesenteric arterial rings were fixed under optimally stretched conditions (see 'Methods' section) for TEM analysis (Figure 5). At low-magnification images (Figure $5 \mathrm{~A}, \mathrm{~B}, \mathrm{C}), 33 \pm 2 \%(n=4)$ of the smooth muscle cells in the organ-cultured arteries with FBS in the absence of Y-27632 were markedly swollen and had reduced cytoplasmic density with fewer organelles including contractile filaments (Figure 5B). No such cells were found in the fresh mesenteric artery (Figure 5A), which suggests that the necrotic smooth muscle cells were induced during the FBS organ culture. On the other hand, most smooth muscle cells in the organ-cultured arteries with $10 \mu \mathrm{M}$ Y-27632 maintained structural integrity of smooth muscle cells except with increased extracellular space (Figure 5C), which corresponds to a mild arterial wall thickening (Figure 2B). At higher magnification images (Figure 5D,E,F,G), the bulk of the cytoplasm was filled with contractile thick and thin filaments and dense bodies in the smooth muscle cells of the mesenteric arteries cultured with $10 \mu \mathrm{M}$ Y-27632 (Figure 5F) as well as those of the fresh mesenteric arteries (Figure 5D). By contrast, in the cultured mesenteric artery without Y-27632, both the contractile filaments and dense bodies were not observed in the swollen and lightly stained cells (Figure 5G, cells marked with an asterisk in Figure 5B), whereas the other darkly stained cells in Figure $5 \mathrm{~B}$ still retained normal thick and thin filaments and dense bodies in the cytoplasm (Figure 5E), as seen in the fresh mesenteric arteries.

\section{Discussion}

In this study, we used an organ culture system of differentiated mesenteric arterial vessels as a physiological model that is reasonably closer to the in vivo environment than the cell culture system. The chronic treatment with the growth stimulant FBS impairs contractility, morphological alteration, and DNA synthesis of arteries during organ culture (Huh et al. 2011; Lindqvist et al. 1999; Murata et al. 2005). To evaluate whether the inhibition of RhoA/ROCK signaling pathway prevents the structural alterations of the artery, we organ-cultured arteries in the presence of ROCK inhibitor Y-27632, together with FBS. Y-27632 prevents the detachment and loss of the endothelial cell layer, the disappearance of contractile thick and thin filaments of smooth muscle cells, and the apoptosis and necrosis during chronic FBS-supplemented organ culture.

According to our previous organ culture study, chronic treatment with Y-27632 protects almost all functional integrities of both endothelial and smooth muscle cells, including excitatory agonist-induced contraction and ACh-induced relaxation (Huh et al. 2011). Y-27632 also successfully inhibited the FBS-induced gradual arterial constriction during organ culture for 7 days. In addition, H-1152, another ROCK inhibitor, and simvastatin, which inhibit RhoA geranylgeranylation (Noma et al. 2006), also showed a significant inhibitory effect on the FBS-induced arterial constriction (Huh et al. 2011). Consistent with those previous works, the present study demonstrated that 

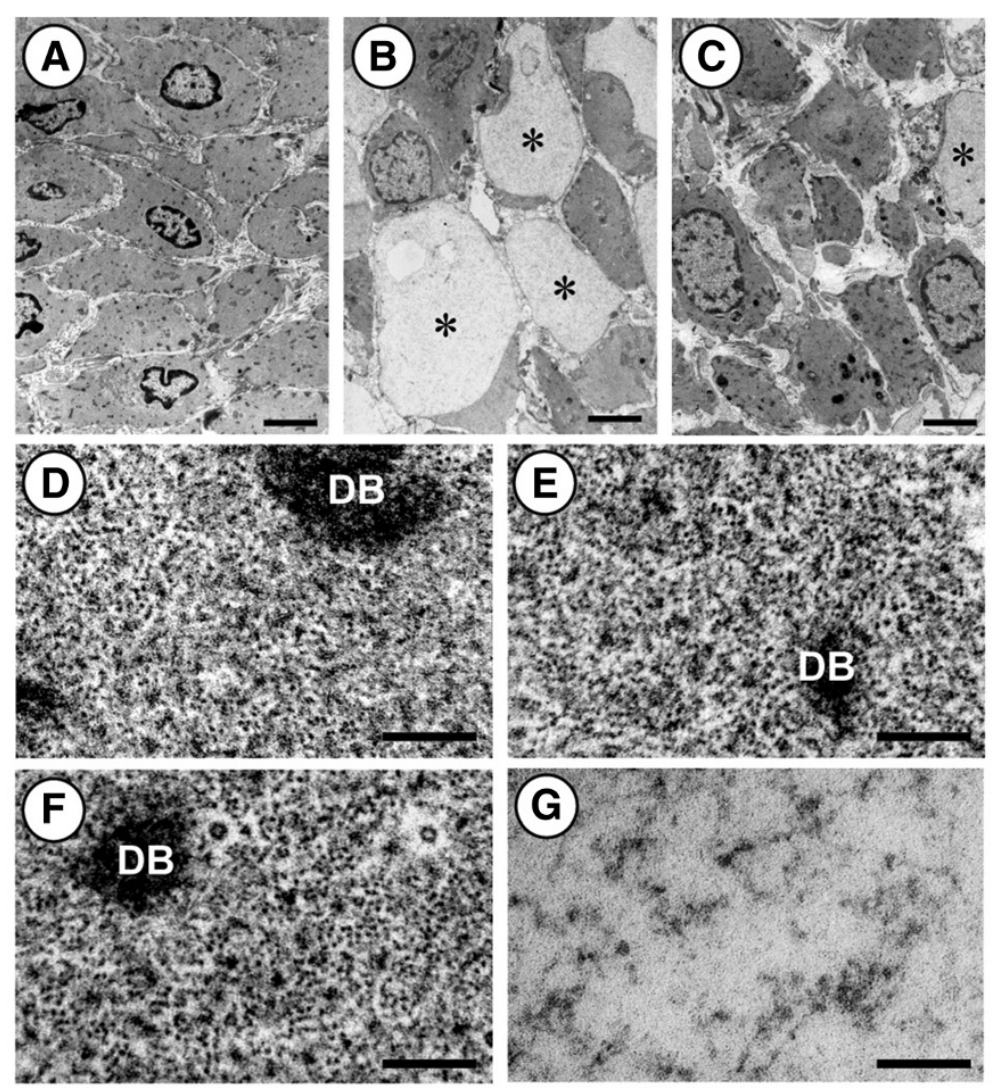

Figure 5 Representative TEM micrographs of cross-sectioned smooth muscle cells from freshly isolated and organ-cultured rabbit mesenteric arteries. Representative low-magnification images of (A) freshly isolated, (B) FBS-supplemented, and (C) FBS with Y-27632supplemented organ-cultured arterial smooth muscle cells, respectively. (D-F) Representative high-magnification images of the darkly stained cells in (A-C), respectively. $\mathbf{( G )}$ A high-magnification image of the lightly stained swollen cells (cells marked with an asterisk in (B)). The asterisk represents the swollen cells in (B) and (C). DB, dense body. Scale bars $=5 \mu \mathrm{m}(\mathbf{A}-\mathbf{C})$ and $200 \mathrm{~nm}$ (D-G).

Y-27632 markedly reduced the morphological alterations in the organ-cultured arterial rings. Interestingly, chronic treatment with Y-27632 did not prevent the FBS-induced overexpression of RhoA and ROCK but rather augmented the expressions to higher levels than those of FBS alone (Huh et al. 2011). Considering that the Y-27632 effectively protects the organ-cultured artery against FBS-induced gradual arterial constriction (Figure 2B), inhibition of the downstream signaling of upregulated RhoA/ROCK may be a major cause for preventing arterial constriction even in higher expression of RhoA and ROCK.

Endothelial cells regulate vascular tone and permeability by producing nitric oxide (NO) (Félétou and Vanhoutte 2006), but the upregulated RhoA/ROCK signaling pathway leads to impaired NO production by suppressing eNOS expression or inhibiting eNOS activity (Noma et al. 2006; Murata et al. 2005; Bolz et al. 2003). Thus, the ROCK inhibitors have been proposed as a therapeutic agent for cardiovascular diseases (Noma et al. 2006; Shimokawa and Takeshita 2005; Loirand et al. 2006). In the case of type 1 diabetes, dysfunction of endothelial cells plays a key role in the pathogenic disorder of vasculature. The upregulation of ROCK and the reduction of the endothelial NO production are closely associated by the involvement of RhoA/ROCK signaling pathway, while suppressing ROCK activity restores vascular function (Cicek et al. 2013; El-Remessy et al. 2010; Kizub et al. 2010; Yao et al. 2013). In the present study, majority of endothelial cell layers were tightly adhered to the elastin layer in the presence of Y-27632, and PECAM expression was also maintained at a level similar to that of the fresh mesenteric artery (Figure 3). These results are consistent with our previous report on the protective role of Y-27632, such as maintenance of eNOS mRNA expression and endothelial cell-mediated relaxation in the organ-cultured mesenteric artery (Huh et al. 2011). Together with our previous report, the present study shows that chronic treatment with Y-27632 protects not only functional but also structural integrities of endothelial cells. Whether Y-27632-induced protection of eNOS mRNA (Huh et al. 2011) and PECAM expression in endothelial cells is caused by or independent 
from maintaining adherence of endothelial cell layers to the smooth muscle cell layers is unclear but warrants examination.

According to recent studies, the inhibition of RhoA/ ROCK signaling pathway promotes the apoptosis of gastric cancer cells (Xiao-Tao et al. 2012). By contrast, inhibition of RhoA/ROCK signaling decreased the neuronal apoptosis in the ischemic penumbra of the rat brain and the penile apoptosis in the cavernous nerve injury model (Wu et al. 2012; Hannan et al. 2013). In the present study, Y-27632 drastically decreased the FBS-induced apoptosis of smooth muscle cells and endothelial cells in the organ-cultured mesenteric artery (Figure 4). This finding may provide another beneficial effect that the inhibition of RhoA/ROCK signaling pathway by Y27632 protects the artery by preventing the apoptosis of both smooth muscle cell and endothelial cell even though the mechanism for ROCK-induced apoptosis needed to be further addressed.

In vascular diseases, the phenotype of smooth muscle cells is easily changed from contractile to synthetic/ proliferative with the loss of contractile filaments in response to various stimuli including growth factors. These phenotypic changes are associated with the downregulation of $\alpha$-actin and h-caldesmon and the upregulation of RhoA and ROCK (Gerthoffer 2007; Owens et al. 2004; Woodsome et al. 2006). In our pervious organ culture study, RhoA and ROCK expression and MYPT1 phosphorylation increased, but the expression of $\alpha$-actin decreased upon chronic FBS treatment. Moreover, the expression of MLC and CPI-17 also decreased to about $50 \%$, both of which have an essential function in the development of vascular smooth muscle contraction (Dimopoulos et al. 2007), but the decrease of MLC and CPI-17 expression was prevented in presence of Y-27632 during organ culture (Huh et al. 2011). To address the effect of the changes in contractile protein expressions on the structural integrity of contractile filaments, we performed the TEM analysis of organcultured arterial smooth muscle cells (Figure 5). The TEM analysis showed that smooth muscle cells in the FBS-supplemented organ culture were markedly necrotized with contractile filament loss (Figure 5B,G), consistent with the previous report by Lindqvist et al. (1999). However, $10 \mu \mathrm{M}$ Y-27632 maintained the structural integrity of the smooth muscle cells with intact actin and myosin filaments (Figure $5 \mathrm{C}, \mathrm{F}$ ). These findings suggest that the overexpression of RhoA and ROCK and the downexpression of $\alpha$-actin, MLC, and CPI-17 are closely related with the loss of contractile actin and myosin filament in organ-cultured smooth muscle cells, as shown in the TEM results of the present study (Figure 5G). In addition, the inhibition of the upregulated RhoA/ROCK signaling pathway and the maintenance of MLC and
CPI-17 expression by the chronic treatment with Y-27632 may play a significant role in the prevention of FBSinduced contractile filament deterioration.

\section{Conclusions}

The findings of the present study provide structural evidence that inactivation of a pathophysiologically important RhoA/ROCK signaling pathway by Y-27632 prevents FBS-supplemented organ culture-induced arterial damages. Y-27632 effectively maintains the arterial diameter and the structural integrity of adhesion of endothelial cells to smooth muscle cells. Also, Y-27632 prevents the apoptosis of both endothelia and smooth muscle cells and the ultrastructural degeneration of contractile actin and myosin filaments in smooth muscle cells. Together, these results suggest that the chronic inhibition of the RhoA/ROCK signaling pathway by inhibitors such as Y-27632 produces preventive effects on FBS-induced vascular complications and could offer a therapeutic means for RhoA/ROCK-mediated vascular diseases, such as hypertension and vasospasm.

\section{Abbreviations}

AT II: Angiotensin II; MLC: Myosin light chain; MYPT1: Myosin phosphatase targeting subunit 1; PECAM: Platelet endothelial cell adhesion molecule; RhoA: Ras homolog gene family member A; ROCK: Rho-associated kinase (Rho-kinase).

\section{Competing interests}

The authors declare that they have no competing interests.

\section{Authors' contributions}

YHH and TK designed research. YHH and TK performed research. YHH, HSK, and TK analyzed data. YHH, HSK, and TK wrote the paper. All authors read and approved the final manuscript.

\section{Acknowledgments}

This research was supported by the National Institute of Health grant R01 HL070881 to TK and the Korea Basic Science Institute grant K32601 to HSK.

\section{Author details}

Division of Electron Microscopic Research, Korea Basic Science Institute, 169-148 Gwahangno, Yuseong-gu, 305-806, Daejeon, Republic of Korea. ${ }^{2}$ Boston Biomedical Research Institute, 64 Grove St, 02472, Watertown, MA, USA. ${ }^{3}$ Department of Microbiology and Physiological Systems, University of Massachusetts Medical School, 55 Lake Avenue North, 01655, Worcester, MA, USA.

Received: 30 May 2013 Accepted: 8 October 2013

Published: 24 Oct 2013

\section{References}

Best PJ, Hasdai D, Sangiorgi G, Schwartz RS, Holmes DR, Simari RD, Lerman A (1999) Apoptosis. Basic concepts and implications in coronary artery disease. Arterioscler Thromb Vasc Biol 19:14-22

Bolz SS, Vogel L, Sollinger D, Derwand R, De Wit C, Loirand G, Pohl U (2003) Nitric oxide-induced decrease in calcium sensitivity of resistance arteries is attributable to activation of the myosin light chain phosphatase and antagonized by the RhoA/Rho kinase pathway. Circulation 107:3081-3087

Brown JH, Del Re DP, Sussman MA (2006) The Rac and Rho hall of fame: a decade of hypertrophic signaling hits. Circ Res 98:730-742

Chen NX, Chen X, O'Neill KD, Atkinson SJ, Moe SM (2010) RhoA/Rho kinase (ROCK) alters fetuin-A uptake and regulates calcification in bovine vascular smooth muscle cells (BVSMC). Am J Physiol Renal Physiol 299:F674-F680 
Cicek FA, Kandilci HB, Turan B (2013) Role of ROCK upregulation in endothelilal and smooth muscle vascular functions in diabetic rat aorta. Cardiovasc Diabetol 12:51, 10.1186/1475-2840-12-51

Dimopoulos G, Semba S, Kitazawa K, Eto M, Kitazawa T (2007) Ca ${ }^{2+}$-dependant rapid $\mathrm{Ca}^{2+}$ sensitization of contraction in arterial smooth muscle. Circ Res 100:121-129

El-Remessy AB, Tawfik FE, Matragoon S, Pillai B, Caldwell RB, Caldwell RW (2010) Peroxynitrite mediates diabetes-induced endothelial dysfunction: possible role of Rho kinase activation. Exp Diabetes Res 2010:247861. doi:10.1155/ 2010/247861

Félétou M, Vanhoutte PM (2006) Endothelial dysfunction: a multifaceted disorder. Am J Physiol Heart Circ Physiol 291:H985-H1002

Gerthoffer WT (2007) Mechanisms of vascular smooth muscle cell migration. Circ Res 100:607-621

Hannan JL, Albersen M, Kutlu O, Gratzke C, Stief CG, Burnett AL, Lysiak JJ, Hedlund P, Bivalacqua TJ (2013) Inhibition of Rho-kinase improves erectile function, increases nitric oxide signaling and decreases penile apoptosis in a rat model of cavernous nerve injury. J Urol 189:1155-1161

Huh YH, Zhou Q, Liao JK, Kitazawa T (2011) ROCK inhibition prevents fetal serum-induced alteration in structure and function of organ-cultured mesenteric artery. J Muscle Res Cell Motil 32:65-76

Kitazawa T, Eto M, Woodsome TP, Khalequzzaman M (2003) Phosphorylation of the myosin phosphatase targeting subunit and $\mathrm{CPI}-17$ during $\mathrm{Ca}^{2+}$ sensitization in rabbit smooth muscle. J Physiol 546:879-889

Kitazawa T, Semba S, Huh YH, Kitazawa K, Eto M (2009) Nitric oxide-induced biphasic mechanism of vascular relaxation via dephosphorylation of CPI-17 and MYPT1. J Physiol 587:3587-3603

Kizub IV, Pavlova OO, Johnson CD, Soloviev Al, Zholos AV (2010) Rho kinase and protein kinase $\mathrm{C}$ involvement in vascular smooth muscle myofilament calcium sensitization in arteries from diabetic rats. Br J Pharmacol 159:1724-1731

Lindqvist A, Nordström I, Malmqvist U, Nordenfelt P, Hellstrand P (1999) Long-term effects of $\mathrm{Ca}^{2+}$ on structure and contractility of vascular smooth muscle. Am J Physiol 277:C64-C73

Loirand G, Guérin P, Pacaud P (2006) Rho kinases in cardiovascular physiology and pathophysiology. Circ Res 98:322-334

Murata T, Suzuki N, Yamawaki H, Sato K, Hori M, Karaki H, Ozaki H (2005) Dexamethasone prevents impairment of endothelium-dependent relaxation in arteries cultured with fetal bovine serum. Eur J Pharmacol 515:134-141

Noma K, Oyama N, Liao JK (2006) Physiological role of ROCKs in the cardiovascular system. Am J Physiol Cell Physiol 290:C661-C668

Owens GK, Kumar MS, Wamhoff BR (2004) Molecular regulation of vascular smooth muscle cell differentiation in development and disease. Physiol Rev 84:767-801

Ozaki H, Karaki H (2002) Organ culture as a useful method for studying the biology of blood vessels and other smooth muscle tissues. Jpn J Pharmacol 89:93-100

Pagiatakis C, Gordon JW, Ehyai S, Mcdermott JC (2012) A novel RhoA/ROCK-CPI17-MEF2C signaling pathway regulates vascular smooth muscle cell gene expression. J Biol Chem 287:8361-8370

Schwartz SM (1997) Smooth muscle migration in atherosclerosis and restenosis. J Clin Invest 99:2814-2816

Shimokawa H, Rashid M (2007) Development of Rho-kinase inhibitors for cardiovascular medicine. Trends in Pharmacol Sci 28:296-302

Shimokawa H, Takeshita A (2005) Rho-kinase is an important therapeutic target in cardiovascular medicine. Arterioscler Thromb Vasc Biol 25:1767-1775

Singh J, Maxwell PJ 4th, Rattan S (2011) Immunocytochemical evidence for PDBu-induced activation of RhoA/ROCK in human internal anal sphincter smooth muscle cells. Am J Physiol Gastrointest Liver Physiol 301:317-325

Somlyo AP, Somlyo AV (2003) $\mathrm{Ca}^{2+}$ sensitivity of smooth muscle and nonmuscle myosin II: modulated by G proteins, kinases, and myosin phosphatase. Physiol Rev 83:1325-1358

Thorne GD, Paul RJ (2003) Effects of organ culture on arterial gene expression and hypoxic relaxation: role of the ryanodine receptor. Am J Physiol Cell Physiol 284:C999-C1005

Uehata M, Ishizaki T, Satoh H, Ono T, Kawahara T, Morishita T, Tamakawa H, Yamagami K, Inui J, Maekawa M, Narumiya S (1997) Calcium sensitization of smooth muscle mediated by a Rho-associated protein kinase in hypertension. Nature 389:990-994

Woodsome TP, Polzin A, Kitazawa K, Eto M, Kitazawa T (2006) Agonist- and depolarization-induced signals for myosin light chain phosphorylation and force generation of cultured vascular smooth muscle cells. J Cell Sci 119:1769-1780
World Health Organization (2011) Global status report on noncommunicable diseases 2010. World Health Organization, Geneva

Wu J, LI J, Hu H, Liu P, Fang Y, Wu D (2012) Rho-kinase inhibitor, fasudil, prevents neuronal apoptosis via the Akt activation and PTEN inactivation in the ischemic penumbra of rat brain. Cell Mol Neurobiol 32:1187-1197

Xiao-Tao X, Qi-Bin S, Yi Y, Bin X, Peng R, Ze-Zhang T (2012) Inhibition of RhoA/ ROCK signaling pathway promotes the apoptosis of gastric cancer cells. Hepatogastroenterology 59:2523-2526

Yao L, Chandra S, Toque HA, Bhatta A, Rojas M, Caldwell RB, Caldwell RW (2013) Prevention of diabetes-induced arginase activation and vascular dysfunction by Rho kinase (ROCK) knockout. Cardiovascular Res 97:509-519

\subsection{6/2093-3371-4-15}

Cite this article as: Huh et al:: ROCK inhibitor, Y-27632, reduces FBS-

induced structural alteration in organ-cultured mesenteric artery. Journal of Analytical Science and Technology 2013, 4:15

\section{Submit your manuscript to a SpringerOpen ${ }^{\odot}$ journal and benefit from:}

- Convenient online submission

- Rigorous peer review

- Immediate publication on acceptance

- Open access: articles freely available online

- High visibility within the field

- Retaining the copyright to your article

Submit your next manuscript at $>$ springeropen.com 